of MEF2C for maintenance of endothelial integrity is also intriguing in light of the recent association of premature coronary artery disease and myocardial infarction with a mutation in the human $M E F 2 A$ gene (20). Since MEF2A is highly expressed in the endothelium and is a substrate for BMK1, it is likely to act within the same EC survival pathway as MEF2C.

A remarkable number of processes within the cardiovascular system are dependent on signaling from MAPKs to MEF2 (7). In addition to its requirement for EC survival, this signaling pathway is important for differentiation and morphogenesis of cardiac and smooth muscle cells, and has been implicated in numerous cardiovascular disorders, including cardiac hypertrophy, dilated cardiomyopathy, coronary artery disease, and myocardial infarction. Further insights into the functions and mechanisms of action of this signaling module promise to provide new opportunities for its therapeutic manipulation in the settings of human disease.

Address correspondence to: Eric N. Olson, Department of Molecular Biology, The University of Texas Southwestern Medical Center at Dallas, 5323 Harry Hines Blvd., NA8.602, Dallas, Texas 75390-9148, USA. Phone: (214) 648-1187; Fax: (214) 648-1196; E-mail: eric.olson@UTSouthwestern.edu.
1. Raman, M., and Cobb, M.H. 2003. MAP kinase modules: many roads home. Curr. Biol. 13:R886-R888.

2. Kasler, H.G., Victoria, J., Duramad, O., and Winoto, A. 2000. ERK5 is a novel type of mitogen-activated protein kinase containing a transcriptional activation domain. Mol. Cell. Biol. 20:8382-8389.

3. Yang, C.C., Ornatsky, O.I., McDermott, J.C., Cruz, T.F., and Prody, C.A. 1998. Interaction of myocyte enhancer factor 2 (MEF2) with a mitogen-activated protein kinase, ERK5/BMK1. Nucleic Acids Res. 26:4771-4777

4. Marinissen, M.J., Chiariello, M., Pallante, M., and Gutkind, J.S. 1999. A network of mitogen-activated protein kinases links $\mathrm{G}$ protein-coupled receptors to the c-jun promoter: a role for c-Jun NH2-terminal kinase, p38, and extracellular signal-regulated kinase 5. Mol. Cell. Biol. 19:4289-4301.

5. Fukuhara, S., Marinissen, M.J., Chiariello, M., and Gutkind, J.S. 2000. Signaling from G protein-coupled receptors to ERK5/Big MAPK 1 involves Galpha $q$ and Galpha 12/13 families of heterotrimeric $G$ proteins. Evidence for the existence of a novel Ras and Rho-independent pathway. J. Biol. Chem. 275:21730-21736.

6. Kato, Y., et al. 2000. Big mitogen-activated protein kinase regulates multiple members of the MEF2 protein family. J. Biol. Chem. 275:18534-18540.

7. McKinsey, T.A., Zhang, C.L., and Olson, E.N. 2002 MEF2: a calcium-dependent regulator of cell division, differentiation and death. Trends Biochem. Sci. 27:40-47.

8. Regan, C.P., et al. 2002. Erk5 null mice display multiple extraembryonic vascular and embryonic cardiovascular defects. Proc. Natl. Acad. Sci. U. S. A. 99:9248-9253.

9. Sohn, S.J., Sarvis, B.K., Cado, D., and Winoto, A 2002. ERK5 MAPK regulates embryonic angiogenesis and acts as a hypoxia-sensitive repressor of vascular endothelial growth factor expression. J. Biol. Chem. 277:43344-43351.

10. Yang, J., et al. 2000. Mekk3 is essential for early embryonic cardiovascular development. Nat. Genet. 24:309-313.
11. Lin, Q., Schwarz, J., Bucana, C., and Olson, E.N. 1997. Control of mouse cardiac morphogenesis and myogenesis by transcription factor MEF2C. Science. 276:1404-1407.

12. Lin, Q., et al. 1998. Requirement of the MADS-box transcription factor MEF2C for vascular development. Development. 125:4565-4574.

13. Bi, W., Drake, C.J., and Schwarz, J.J. 1999. The transcription factor MEF2C-null mouse exhibits complex vascular malformations and reduced cardiac expression of angiopoietin 1 and VEGF. Dev. Biol. 211:255-267.

14. Hayashi, M., et al. 2004. Targeted deletion of $B M K 1 / E R K 5$ in adult mice perturbs vascular integrity and leads to endothelial failure. J. Clin. Invest. 113:1138-1148. doi:10.1172/JCI200419890.

15. Liu, L., et al. 2003. ERK5 activation of MEF2-mediated gene expression plays a critical role in BDNFpromoted survival of developing but not mature cortical neurons. Proc. Natl. Acad. Sci. U. S. A. 100:8532-8537

16. Linseman, D.A., et al. 2003. Inactivation of the myocyte enhancer factor-2 repressor histone deacetylase- 5 by endogenous $\mathrm{Ca}^{2+} / /$ calmodulindependent kinase II promotes depolarization-mediated cerebellar granule neuron survival. J. Biol. Chem. 278:41472-41481.

17. Mao, Z., Bonni, A., Xia, F., Nadal-Vicens, M., and Greenberg, M.E. 1999. Neuronal activity-dependent cell survival mediated by transcription factor MEF2. Science. 286:785-790.

18. Li, M., et al. 2001. Myocyte enhancer factor $2 \mathrm{~A}$ and $2 \mathrm{D}$ undergo phosphorylation and caspase-mediated degradation during apoptosis of rat cerebellar granule neurons. J. Neurosci. 21:6544-6552.

19. Pi, X., Yan, C., and Berk, B.C. 2004. Big mitogenactivated protein kinase (BMK1)/ERK5 protects endothelial cells from apoptosis. Circ. Res. 94:362-369.

20. Wang, L., Fan, C., Topol, S.E., Topol, E.J., and Wang, Q. 2003. Mutation of MEF2A in an inherited disorder with features of coronary artery disease. Science. 302:1578-1581.

\title{
Insig: a significant integrator of nutrient and hormonal signals
}

\author{
Alan D. Attie \\ Department of Biochemistry, University of Wisconsin-Madison, Madison, Wisconsin, USA.
}

\begin{abstract}
Lipogenesis is regulated by sterols and by insulin through the regulated expression and activation of the sterol regulatory element-binding proteins (SREBPs). A new study shows one way in which sterol and insulin regulation can be decoupled (see the related article beginning on page 1168). In transgenic mice overexpressing a protein that regulates SREBP activation, lipogenesis is more sensitive to cholesterol and less sensitive to insulin.
\end{abstract}

\author{
Nonstandard abbreviations used: insulin receptor \\ substrate-2 (IRS-2); SREBP cleavage-activating protein \\ (SCAP); sterol regulatory element-binding \\ protein/adipocyte differentiation factor-1 \\ (SREBP/ADD1). \\ Conflict of interest: The author has declared that no \\ conflict of interest exists. \\ Citation for this article: \\ J. Clin. Invest. 113:1112-1114 (2004). \\ doi:10.1172/JCI200421450.
}

Animal cells, like nations, have a domestic and a foreign policy. "Domestic policy" involves local control of metabolism, responses to energy charge, redox status, or the concentration of key allosteric regulators (e.g., malonyl-CoA and fructose 2,6-bisphosphate). The world of a multicellular organism imposes a "foreign policy" on its cells. They must respond to global signals in the interest of the whole organism.

Global and local signals invariably converge (as former Congressman Tip O'Neil famously quipped, "All politics is local."). Lipogenesis is a case in point. Feeding, fasting, and refeeding cause large changes in the rates of fatty acid and cholesterol synthesis. These changes are evoked by both the carbohydrate and the lipid components of the diet. We now have a molecular window into some of the key molecules that integrate nutrient-derived signals.

A key breakthrough was the simultaneous discovery by Tontonoz et al. (1) and Yokoyama et al. (2) of the sterol regulatory ele- 


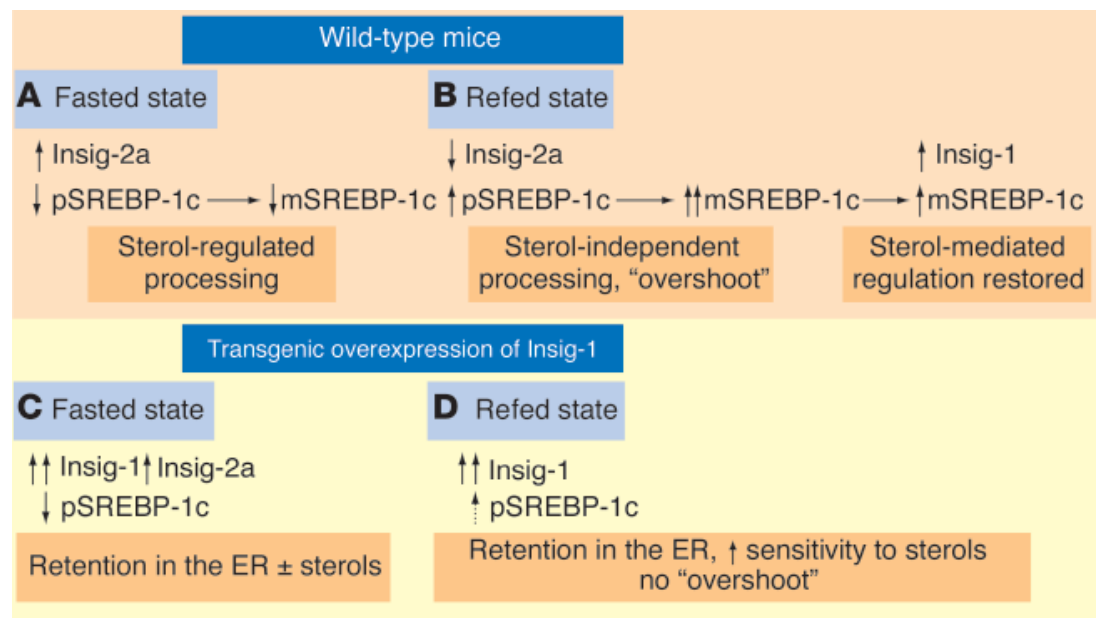

\section{Figure 1}

Integration of metabolic signals at the ER membrane. (A) During fasting, expression of the SREBP-1c precursor (pSREBP-1c) is reduced. Insig-2a is expressed and binds to SCAP, causing retention of a very low amount of pSREBP-1c in the ER in the presence of sterols. (B) Upon refeeding, insulin increases the expression of pSREBP-1c and decreases the expression of Insig-2a. The low abundance of both Insig-1 and Insig-2a leads to greatly increased processing of pSREBP-1c to mature SREBP-1c (mSREBP-1c), leading to a lipogenesis rate that is more than tenfold higher than that of the basal state, which represents an "overshoot." The increased level of mSREBP-1c promotes expression of Insig-1. At a critical stoichiometry relative to SCAP, Insig-1 restores sterol-mediated regulation of pSREBP-1c processing. The lipogenesis rate then returns to the original basal level. (C) With transgenic overexpression of Insig-1, the cycling of lipogenesis between the fed and fasted states is dampened. The high ratio of Insig-1 to SCAP causes retention of pSREBP-1c in the ER. Fasted mice have a modest additional drop in lipogenesis due to the reduction of pSREBP-1c expression and increased Insig-2a levels. (D) With refeeding, the transgenic mice express extremely low levels of pSREBP-1c due to their inability to produce enough mSREBP-1c to drive the transcription of the $p S R E B P-1 c$ gene. The persistently elevated level of Insig-1 abolishes the dramatic "overshoot" in lipogenesis seen in the wildtype refed mice but still mediates an enhanced sensitivity of pSREBP-1c processing to sterols.

ment-binding protein/adipocyte differentiation factor-1 (SREBP/ADD1). The Brown and Goldstein laboratory went on to show that in the liver, two isoforms, SREBP-1c and SREBP-2, are master transcriptional regulators of the fatty acid and cholesterol synthesis pathways, respectively (3). The SREBPs reside as inactive precursors in the ER membrane. Upon vesicular transport to the Golgi, they are proteolytically cleaved to liberate a fragment that enters the nucleus and activates SRE-containing genes. The transport step is blocked by sterols. This defines the ER membrane as a locus wherein proteins sense lipids and act as the gatekeepers of SREBPs.

What are the protein gatekeepers? Through somatic cell genetic studies of mutant cell lines with defective feedback regulation of sterol synthesis, Brown and Goldstein discovered an SREBP escort protein, SREBP cleavage-activating protein (SCAP). SCAP has a sterol-sensing domain that is necessary for sterols to inhibit SREBP transport to the Golgi (4). Without SCAP, cells require exogenous cholesterol to survive.
Gene dosage studies of SCAP in hamster cells suggested that an additional factor could be titrated to abolish the sterol responsiveness of SREBP processing (5). Coimmunoprecipitation studies led to the discovery of a SCAP-interacting protein, Insig-1 (6). A homologous gene, Insig-2, was also identified (7). Careful gene dosage studies of Insig1 showed that only within a narrow window of the Insig-1/SCAP stoichiometry does the system respond to sterol regulation. When SCAP is in molar excess relative to Insig-1, SREBP processing is insensitive to sterols. With a large excess of Insig-1, SREBP is retained in the ER, even in the absence of sterols. This has led researchers to ask whether these titration studies extend to the liver of an intact animal.

\section{Insig-1 overexpression suppresses lipogenesis in vivo}

In this issue of the JCI, Engelking and coworkers show that hepatic overexpression of Insig-1 in transgenic mice caused a marked reduction in nuclear SREBPs, which further decreased when mice were fed a high-cholesterol diet (8). Thus, the in vitro titration experiments correctly predicted the effect of Insig- 1 on the lipogenesis rate in vivo. The in vivo effects of cholesterol on expression of SREBP are complicated by the fact that sterols activate SREBP-1c (not SREBP-2) gene expression through the liver $\mathrm{X}$ receptor transcription factors (9). Thus, at low levels, dietary cholesterol decreases the level of mature SREBP-1c protein, but at higher levels, it induces SREBP-1c gene expression (8).

\section{Insig-1 overexpression reduces the sensitivity of lipogenesis to insulin}

The first link between SREBP and insulin came from the discoveries that insulin increases the expression of SREBP-1c (10, $11)$, that transgenic mice overexpressing SREBP-1c do not suppress lipogenesis in response to fasting (12), and that lipogenesis is not induced in mice lacking SREBP-1c when they are fed a high-carbohydrate diet (13). Given that lipogenesis is regulated by insulin, inevitable questions arise: How do cells integrate regulation of lipogenesis by insulin with regulation by lipids? What molecules "sense" lipid and hormonal status? When they act together, which signals are dominant?

Normally, fasting causes a downregulation of SREBP- $1 \mathrm{c}$ and thus a reduction in mature SREBP-1c. Refeeding leads to an induction of SREBP-1c and Insig-1 and greatly increased levels of mature SREBP-1c (Figure 1). Surprisingly, transgenic overexpression of Insig-1 blunted the insulin response; refeeding produced only a modest increase in SREBP-1c (8). In contrast, the sensitivity of SREBP-1c processing to cholesterol was enhanced.

Insulin cycling during the transition from feeding to fasting causes the liver to switch from glycolysis/lipogenesis to gluconeogenesis/ketogenesis. After refeeding, insulin suppresses the expression of insulin receptor substrate-2 (IRS-2) and phosphoenolpyruvate carboxykinase, resulting in decreased gluconeogenesis $(14,15)$. This pathway was intact in the Insig-1 transgenic mice (8).

Left undefined is the role of Insig- $2 \mathrm{a}$, a liver-specific isoform of Insig-2. Like Insig-1, Insig-2a causes retention of SREBP in the ER; however, it does so only in the presence of a concentration of sterols (7). Unlike Insig-1, Insig-2a is suppressed by insulin and induced by fasting. Early in the transition from fasting to feeding, Insig-1 levels are low and Insig-2a expression is repressed, allowing the insulin-induced SREBP-1c protein to be processed. Increased SREBP-1c induces Insig-1 
expression and restores sterol sensing (Figure 1). Upon fasting, Insig-2a levels rise, suppressing lipogenesis in a sterol-dependent fashion. Insulin cycling might be critical for the prevention of excessive lipogenesis. It creates a brief window during refeeding when Insig levels begin to decline and there is insufficient Insig-1 to suppress lipogenesis. However, with induction of Insig-1, lipogenesis is held in check. Thus, a wild-type refed animal "overshoots" its rate of lipogenesis (7). In contrast, transgenic mice overexpressing Insig-1 effectively abolish the cycling of Insig-1 expression and therefore do not overshoot lipogenesis upon refeeding (Figure 1). This leads to a dramatic $80 \%$ reduction in plasma triglycerides in the refed mice (8).

\section{Potential implications for insulin resistance}

With chronic hyperinsulinemia, the normally converse relationship between lipogenesis and gluconeogenesis can be disrupted (16). This occurs in models of leptin deficiency (e.g., the leptin ${ }^{\mathrm{ob}}$ mutation or congenital lipodystrophy) or leptin resistance. Deletion of IRS-2 leads to leptin resistance (17), suggesting a convergence between the leptin and insulin signaling pathways. Leptin resistance boosts lipogenesis in the liver through increased SREBP-1c. Deletion of SREBP-1c reduces the rate of lipogenesis of leptin-deficient animals but does not reverse insulin resistance, hence other aspects of leptin signaling influence insulin signaling (18).
The focus of the Insig story will likely turn to Insig-2a. Is Insig-2a affected by leptin deficiency or leptin resistance? What happens to Insig-2a under conditions of chronic hyperinsulinemia? We hope to learn why two closely related proteins are oppositely regulated by insulin.

Address correspondence to: Alan D. Attie, Department of Biochemistry, University of Wisconsin-Madison, 433 Babcock Drive, Madison, Wisconsin 53706, USA. Phone: (608) 262-1372; Fax: (608) 263-9609; E-mail: attie@biochem.wisc.edu.

1. Tontonoz, P., Kim, J.B., Graves, R.A., and Spiegelman, B.M. 1993. ADD1: a novel helix-loop-helix transcription factor associated with adipocyte determination and differentiation. Mol. Cell. Biol. 13:4753-4759.

2. Yokoyama, C., et al. 1993. SREBP-1, a basic-helixloop-helix-leucine zipper protein that controls transcription of the low density lipoprotein receptor gene. Cell. 75:187-197.

3. Horton, J.D., Goldstein, J.L., and Brown, M.S. 2002 SREBPS: activators of the complete program of cholesterol and fatty acid synthesis in the liver. J. Clin. Invest. 109:1125-1131. doi:10.1172/JCI200215593.

4. Hua, X., Sakai, J., Brown, M.S., and Goldstein, J.L. 1996. Regulated cleavage of sterol regulatory element binding proteins requires sequences on both sides of the endoplasmic reticulum membrane. J. Biol. Chem. 271:10379-10384.

5. Yang, T., Goldstein, J.L., and Brown, M.S. 2000. Overexpression of membrane domain of SCAP prevents sterols from inhibiting SCAP.SREBP exit from endoplasmic reticulum. J. Biol. Chem. 275:29881-29886.

6. Yang, T., et al. 2002. Crucial step in cholesterol homeostasis: sterols promote binding of SCAP to INSIG-1, a membrane protein that facilitates retention of SREBPs in ER. Cell. 110:489-500.

7. Yabe, D., Brown, M.S., and Goldstein, J.L. 2002 Insig-2, a second endoplasmic reticulum protein that binds SCAP and blocks export of sterol regulatory element-binding proteins. Proc. Natl. Acad. Sci. U. S. A. 99:12753-12758.

8. Engelking, L.J., et al. 2004. Overexpression of Insig-1 in the livers of transgenic mice inhibits SREBP processing and reduces insulin-stimulated lipogenesis. J. Clin. Invest. 113:1168-1175. doi:10.1172/JCI200420978.

9. Repa, J.J., et al. 2000. Regulation of mouse sterol regulatory element-binding protein-1c gene (SREBP-1c) by oxysterol receptors, LXRalpha and LXRbeta. Genes Dev. 14:2819-2830.

10. Foretz, M., et al. 1999. ADD1/SREBP-1c is required in the activation of hepatic lipogenic gene expression by glucose. Mol. Cell. Biol. 19:3760-3768.

11. Shimomura, I., et al. 1999. Insulin selectively increases SREBP-1c mRNA in the livers of rats with streptozotocin-induced diabetes. Proc. Natl. Acad. Sci. U. S. A. 96:13656-13661.

12. Shimano, H., et al. 1997. Isoform 1c of sterol regulatory element binding protein is less active than isoform 1a in livers of transgenic mice and in cultured cells. J. Clin. Invest. 99:846-854.

13. Liang, G., et al. 2002. Diminished hepatic response to fasting/refeeding and liver $\mathrm{X}$ receptor agonists in mice with selective deficiency of sterol regulatory elementbinding protein-1c. J. Biol. Chem. 277:9520-9528.

14. Zhang, J., et al. 2001. Insulin inhibits transcription of IRS-2 gene in rat liver through an insulin response element (IRE) that resembles IREs of other insulin-repressed genes. Proc. Natl. Acad. Sci. U. S. A. 98:3756-3761.

15. Puigserver, P., et al. 2003. Insulin-regulated hepatic gluconeogenesis through FOXO1-PGC-1alpha interaction. Nature. 423:550-555.

16. Shimomura, I., et al. 2000. Decreased IRS-2 and increased SREBP-1c lead to mixed insulin resistance and sensitivity in livers of lipodystrophic and ob/ob mice. Mol. Cell. 6:77-86.

17. Tobe, K., et al. 2001. Increased expression of the sterol regulatory element-binding protein-1 gene in insulin receptor substrate-2/- mouse liver. J. Biol. Chem. 276:38337-38340.

18. Yahagi, N., et al. 2002. Absence of sterol regulatory element-binding protein-1 (SREBP-1) ameliorates fatty livers but not obesity or insulin resistance in Lep $^{\mathrm{ob}} /$ Lep $^{\mathrm{ob}}$ mice. J. Biol. Chem. 277:19353-19357.

\title{
Experimental autoimmune hearing loss
}

\author{
Peter Billings
}

Division of Otolaryngology—Head and Neck Surgery, University of California, San Diego, and Research Service of the Department of Veterans Affairs, San Diego, California, USA.

\begin{abstract}
Understanding of autoimmune sensorineural hearing loss (ASNHL) has been hindered by the inaccessibility of the inner ear to biopsy and the lack of workable animal models. A report in this issue of the JCI describes a mouse model of $\mathrm{CD}^{+} \mathrm{T}$ cell-mediated ASNHL induced by immunization with peptides from the inner ear-specific proteins cochlin and $\beta$-tectorin (see the related article beginning on page 1210).
\end{abstract}

\author{
Nonstandard abbreviations used: antigen (Ag); \\ autoimmune inner ear disease (AIED); autoimmune \\ sensorineural hearing loss (ASNHL); inner ear (IE); \\ sensorineural hearing loss (SNHL). \\ Conflict of interest: The author has declared that no \\ conflict of interest exists. \\ Citation for this article: \\ J. Clin. Invest. 113:1114-1117 (2004). \\ doi:10.1172/JCI200421632.
}

The inner ear (IE), like most other specialized tissues and organs, can become the target of an autoimmune attack. Sensorineural hearing loss (SNHL) is often an early, although presumably secondary, complication of various non-organ-specific autoimmune diseases; however, the IE can also represent the primary focus of a unique disease entity, autoimmune IE disease (AIED) (1). Fortunately the disease is rare, but the small population size of affected individuals and the inaccessibility of the IE during an acute attack have hindered progress in our understanding of the etiology, diagnosis, and treatment of this disease. AIED is diagnosed by exclusion of other disorders that mimic it. The hearing loss is typically bilateral, asymmetric, and fluctuating and deteriorates rapidly over weeks or months; balance and equilibrium may or may not be affected. Diagnosis of AIED is tentatively confirmed if there is a positive response to trial corticosteroid 